\title{
Psicopatología en el cine paraguayo: una primera aproximación
}

\author{
Psychopathology in Paraguayan cinema: a first approach
}

Matías Franco-Di Giuseppe $\mathbb{1}^{1}$, Gimena Beatriz Hidalgo-Hidalgo@1, Micaela de los Milagros Martinsevich ${ }^{1}$, Juan Víctor Fabián Mendoza-Morel@1, Gissell Elizabeth Meza-Espinoza@1, Juan Sebastián Ochipinti-Agüero@1,

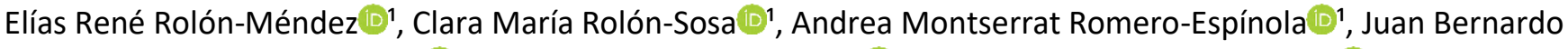
Ruiz Díaz-Garrido@', César Iván Ruiz Díaz-Insfrán@1, Laura Amelia Sanabria-Cañete@1, Gladys Matilde Torres-Candia $\mathbb{1}^{1}$, Oscar García ${ }^{1}$, Julio Torales ${ }^{1}$

${ }^{1}$ Universidad Nacional de Asunción, Facultad de Ciencias Médicas, San Lorenzo, Paraguay.

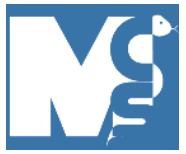

Recibido: 05/11/2020

Revisado: 07/11/2020

Aceptado:03/12/2020

\section{Autor correspondiente}

Julio Torales

Universidad Nacional de Asunción

San Lorenzo - Paraguay

jtorales@med.una.py

\section{Conflictos de interés}

Los autores declaran no poseer conflictos de interés.

\section{Fuente de financiación}

Los autores no recibieron apoyo financiero para la investigación, autoría y/o publicación de este artículo.

Este artículo es publicado bajo una licencia de Creative Commons Reconocimiento 4.0 Internacional.

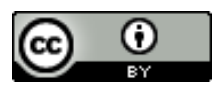

\section{RESUMEN}

Introducción: el cine puede ser una herramienta útil para la educación médica. En la Psiquiatría, específicamente, el cine permite explorar las representaciones de diferentes trastornos mentales, cómo es vivir con una enfermedad mental, el estigma, la ética médica, la relación psiquiatra/paciente, entre otros. El objetivo de este estudio fue determinar la presencia de algún tipo de psicopatología en películas producidas en Paraguay, con el fin de que las mismas puedan ser utilizadas como herramientas didácticas en la enseñanza de la Psicopatología. Metodología: el diseño de esta investigación fue de tipo cualitativo exploratorio. Se seleccionaron, por conveniencia, 10 películas paraguayas, producidas entre los años 2010 y 2019. Las películas se eligieron tomando en cuenta su disponibilidad de visualización en las plataformas Netflix, Amazon Prime Video, HBO GO, Apple TV+, YouTube, y en formato DVD. Resultados: se analizaron en busca de Psicopatología las siguientes películas Universo Servilleta (2010), Cuchillo de Palo (2010), 7 cajas (2012), La Enamorada (2012), Latas Vacías (2014), La Chiperita (2015), Los Buscadores (2017), Leal (2018), Las Herederas (2018), y El Supremo Manuscrito (2019). En las películas seleccionadas se encontraron personajes con signos y síntomas que pueden resultar útiles para enseñar algunos de los principales síndromes psiquiátricos: trastornos depresivos, ansiedad, intentos de suicidio, rasgos obsesivo-compulsivos, trastornos de la personalidad, ideas delirantes, alteraciones del control de los impulsos y adicciones. Conclusión: el cine paraguayo, cada vez más rico y diverso, además de ser una fuente de inspiración y entretenimiento, puede ayudar en la enseñanza de la Medicina en general, y de la Psiquiatría en particular.

Palabras clave: cine; educación médica; Psiquiatría; trastornos mentales; Psicopatología

\section{ABSTRACT}

Introduction: Cinema can be a useful tool for medical education. In Psychiatry, specifically, cinema allows us to explore representations of different mental disorders, what it is like to live with mental illness, stigma, medical ethics, the psychiatrist/patient relationship, among others. The objective of this study was to determine the presence of some type of psychopathology in films produced in Paraguay, so that they can be used as didactic tools in the teaching of Psychopathology. Methodology: The design of this research was qualitative and exploratory. For convenience, 10 Paraguayan films, produced between 2010 and 2019, were selected. The films were chosen taking into account their availability for viewing on Netflix, Amazon Prime Video, HBO GO, Apple TV+, and YouTube platforms, and in DVD format. Results: The following films were analyzed in search of psychopathology Universo Servilleta (2010), Cuchillo de Palo (2010), 7 cajas (2012), La Enamorada (2012), Latas Vacías (2014), La Chiperita (2015), Los Buscadores (2017), Leal (2018), Las Herederas (2018), and El Supremo Manuscrito (2019). In the selected films, characters with signs and symptoms that may be useful to teach some of the main psychiatric syndromes were found: depressive disorders, anxiety, suicide attempts, obsessive-compulsive traits, personality disorders, delusions, impulse control disorders, and addictions. Conclusion: Paraguayan cinema, increasingly rich and diverse, in addition to being a source of inspiration and entertainment, can help in the teaching of Medicine in general, and Psychiatry in particular.

Keywords: cinema; medical education; Psychiatry; mental disorders; Psychopathology. 


\section{INTRODUCCIÓN}

El cine posee la capacidad de hacernos partícipes de las innumerables posibilidades creativas e imaginativas que tenemos los seres humanos. Combinando imágenes, sonido y movimiento, puede servir para mostrar lo más sublime de la mente y la conducta humana, aunque también puede dejar en evidencia lo más terrible (1). A decir de Bhugra (2), el cine juega un papel importante en la psique social, política y cultural de nuestro mundo $y$, añadimos, también en la educacional.

En ese sentido, el cine puede ser una herramienta útil para la educación médica. En la Psiquiatría, específicamente, el cine permite explorar las representaciones de diferentes trastornos mentales, cómo es vivir con una enfermedad mental, el estigma, la ética médica, la relación psiquiatra/paciente, entre otros (3).

A lo largo de las décadas, las películas han representado diversas afecciones psiquiátricas que van desde el suicidio y los trastornos por uso de sustancias hasta los trastornos de personalidad y la esquizofrenia. Aunque estas representaciones no siempre sean exactas, pueden ser lo suficientemente buenas para que comprendamos y analicemos algún aspecto del trastorno. Definitivamente, los tiempos han cambiado. Antes las películas eran criticadas por la descripción negativa que hacían de los médicos psiquiatras y de los trastornos mentales, mientras que hoy día, en los tiempos actuales, las mismas pueden ser utilizadas para enseñar en las aulas.

Las películas se constituyen en buenas herramientas de enseñanza, porque a través de un lenguaje menos técnico se hace que el aprendizaje de la Psiquiatría sea más placentero que aquel proveniente de clases tradicionales. Además, los pacientes con síntomas psicopatológicos específicos no siempre están disponibles para ser evaluados por parte de médicos en formación o estudiantes de Medicina, por lo que las películas pueden suplir en parte esa ausencia de vivencia práctica, además de evitar los conflictos éticos y de derechos humanos que pueden surgir al exponer a una persona con un trastorno mental a grupos grandes de personas durante las clases prácticas impartidas en las Facultades de Medicina. Aquí se debe destacar que las escenas cinematográficas no sustituyen la anamnesis y el examen de una persona con un trastorno mental, pero pueden ayudar, especialmente a estudiantes y a médicos jóvenes, en la identificación de los principales signos y síntomas en Psiquiatría (4).
Tomando en cuenta lo anterior, esta breve investigación buscó determinar la presencia de algún tipo de psicopatología en películas producidas en Paraguay, con el fin de que las mismas puedan ser utilizadas como herramientas didácticas en la enseñanza de la Psicopatología a estudiantes y profesionales de la Medicina, Psicología y Trabajo Social, entre otros.

\section{METODOLOGÍA}

El diseño de esta investigación fue de tipo cualitativo exploratorio. Se seleccionaron, por conveniencia, 10 películas paraguayas, producidas entre los años 2010 y 2019. Las películas se eligieron tomando en cuenta su disponibilidad de visualización en las plataformas Netflix, Amazon Prime Video, HBO GO, Apple TV+, YouTube, y en formato DVD.

Luego de ver cada una de las películas, las mismas se ordenaron según el año de producción, género, director, actores y psicopatología encontrada. El tipo de psicopatología fue determinado según los criterios diagnósticos de la $5 .^{a}$ edición del Manual Diagnóstico y Estadístico de los Trastornos Mentales (DSM-5) de la Asociación Americana de Psiquiatría (5).

Este estudio se enmarcó en la línea de investigación "Epidemiología y psicopatología de los trastornos mentales" (instalada por Resolución D. N. ${ }^{\circ} 1950 / 2017$ del Decano de la Facultad de Ciencias Médicas de la Universidad Nacional de Asunción) y fue aprobado por la Cátedra de Psicología Médica de la Facultad de Ciencias Médicas de la Universidad Nacional de Asunción, Paraguay. Se respetaron los principios enumerados en la Declaración de Helsinki. La existencia de alteraciones psicopatológicas se hizo analizando las características y conductas de los personajes de las películas incluidas, y no desde una perspectiva que incluya algún aspecto personal de los actores $\mathrm{o}$ actrices

\section{RESULTADOS}

Se analizaron en busca de psicopatología las siguientes películas Universo Servilleta (2010), Cuchillo de Palo (2010), 7 cajas (2012), La Enamorada (2012), Latas Vacías (2014), La Chiperita (2015), Los Buscadores (2017), Leal (2018), Las Herederas (2018), y El Supremo Manuscrito (2019).

En la presente investigación, las películas seleccionadas presentan personajes con signos y síntomas que pueden resultar útiles para enseñar algunos de los principales síndromes psiquiátricos: trastornos depresivos, ansiedad, intentos de suicidio, rasgos 
obsesivo-compulsivos, trastornos de la personalidad, ideas delirantes, alteraciones del control de los impulsos y adicciones. Los hallazgos psicopatológicos y otros datos de las películas pueden puede verse en la tabla 1.

TABLA 1. PSICOPATOLOGÍA EN PELÍCULAS PARAGUAYAS

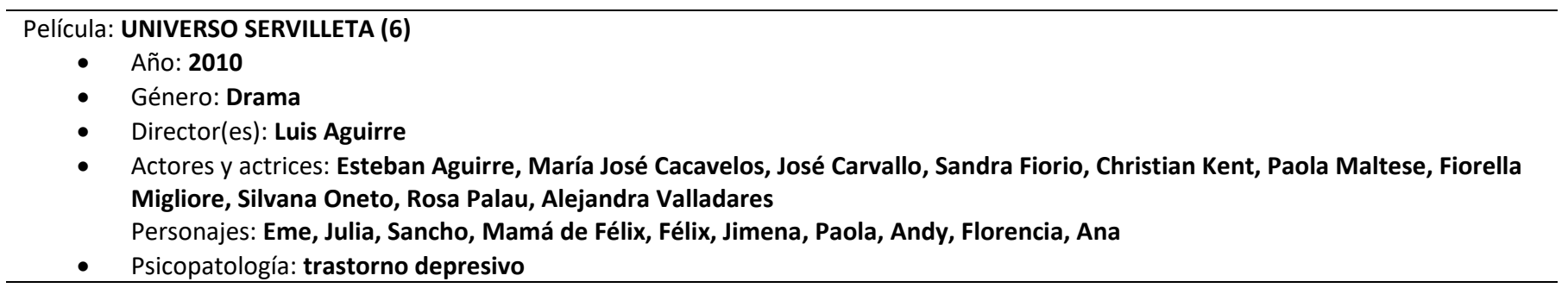

Película: CUCHILLO DE PALO (7)

- Año: 2010

- Género: Documental

- Director(es): Renate Costa

- Actores y actrices (como ellos mismos): Rodolfo Costa, Pedro Costa, Renate Costa, Manuel Cuenca, Miguel Auad (Petunia), Nancy Baruja, Liz Paola, Anónimo

- Psicopatología: trastorno depresivo, estrés postraumático, trastorno general de la personalidad

Película: 7 CAJAS (8)

- Año: 2012

- Género: Drama, crimen, aventura

- Director(es): Juan Carlos Maneglia, Tana Schémbori

- Actores y actrices: Celso Franco, Víctor Sosa, Lali Gonzalez, Nico García, Miguel “Paletita” Romero, Manu Portillo, Mario Toñanez, Nelly Davalos, Roberto Cardozo, Jin Hyuk “Johnny” Kim, Luís Gutiérrez, Liliana Álvarez, Katia García, Atil Closs, Junior Rodríguez, Stephen Jang

Personajes: Nelson, Liz, Luis, Darío, Oficial Servián, Sargento Osorio, Tamara, Gus, Jorge, Alejandra, Leti Sánchez, Esteban, Tano, Don Chan

- Psicopatología: alteraciones del control de los impulsos y de la conducta, trastorno de la comunicación y del lenguaje, disociación

Película: LA ENAMORADA (9)

- Año: 2012

- Género: Drama, romance

- Director(es): Miguel Martín Crespo

- Actores y actrices: Marcela Achinelli, Natalia Alvarenga, Selva Fox, Christian Kent, Daniel Milesi, Silvio Rodas

- Personajes: Victoria, Don Zoilo, Dr. Juan Bautista, Madre de Victoria

- Psicopatología: ansiedad, obsesiones-compulsiones

Película: LATAS VACÍAS (10)

- Año: 2014

- Género: Drama, suspenso

- Director(es): Hérib Godoy

- Actores y actrices: Aníbal Ortiz, Antonia Florentín Medina, Máximo Florentín

- Personajes: Alfonso, Verónica, Atilio

- Psicopatología: trastorno depresivo, ideas delirantes de persecución y de referencia

Película: LA CHIPERITA (11)

- Año: 2015

- Género: Drama

- Director(es): Hugo Cataldo

- Actores y actrices: Patricia Paredes, Bruno Sosa, Hebe Duarte, María Liz Rojas, Giannina Lezcano

- Personajes: Virgilia, Walter, Candelaria, Juanita, Vero

- Psicopatología: pánico, rasgos de personalidad de tipo paranoide

Película: LOS BUSCADORES (12)

- $\quad$ Año: 2017

- Género: Aventura, comedia

- Director(es): Juan Carlos Maneglia, Tana Schémbori

- $\quad$ Actores y actrices: Tomás Arredondo, Christian Ferreira, Mario Toñánez, Cecilia Torres, Sandra Sanabria, Leticia Sosa, José Pérez, Amada Gómez, Nelly Dávalos, Martín Oviedo, José Fernández, Angélica Macuacua, Gustavo Cabaña, Rodrigo Caballero, Mario González, Luis Troche, Miguel Romero

- Personajes: Manu, Fito, Don Elio, llu, Lili, Lore, Abuela de Manu, Madre de Manu, Beto, José Ortiz, Esposa del Embajador, Cliente del cibercafé, Julio, Hermano de llu, Guardia 1, Primer buscador 
Película: LEAL, SOLO HAY UNA FORMA DE VIVIR (13)

- $\quad$ Año: 2018

- Género: Acción, aventura

- Director(es): Rodrigo Salomón, Pietro Scappini

- $\quad$ Actores y actrices: Luis Aguirre, Fini Bocchino, Bruno Sosa Bofinger, Fabio Chamorro, Dani Da Rosa, José Roberto Fretes Romero, Andrea Frigerio. David Gerber, Ana Maria Imizcoz, Mauricio A. Jortack, Félix Alberto Medina Ortiz, Alcides Oviedo Cáceres, Andrea Quattrocchi, Sergio Quiñonez Román, Silvio Rodas, Rafael Rojas Doria, Juan Cruz Rojas, Gonzalo Vivanco, Roberto Weiss

- Personajes: Gorostiaga, María José, Dante, Chamorro, Espínola, Sebastián Assad, Ángeles, Ruso, Elvira, Roberto Vallejos, Ibáñez, Capote, Betty, Tembleque, Ministro Fernández, Papapa, José Assad, Salcedo, Fariña

- Psicopatología: adicciones, rasgos de personalidad narcisista y de personalidad antisocial

Película: LAS HEREDERAS (14)

- Año: 2018

- Género: Drama, cine LGBT

- Director(es): Marcelo Martinessi

- Actores y actrices: Ana Brun, Margarita Irún, Ana Ivanova, Nilda González, María Martins, Alicia Guerra

- Personajes: Chela, Chiquita, Angy, Pati, Pituca, Carmela

- Psicopatología: trastorno depresivo, obsesiones-compulsiones

Película: EL SUPREMO MANUSCRITO (15)

- Año: 2019

- Género: Drama, suspenso

- $\quad$ Director(es): Jorge Diaz de Bedoya, Michael Kovich Jr.

- Actores y actrices: Fernando Abadie, Sandra Guillén, Rafael Alfaro, Ricardo Dalmacci, Javier Enciso, Andrea Quattrocchi, Sary López, Nathan Haase, Katia Acosta, Claudia Scavone

- Personajes: Anton Remianiuk, Ana Morel, El doctor, El subastador, Tateti, Valeria Aguirre, Camila Agente Darren, Rufina Mendoza, Fiscal Amalia

- Psicopatología: estrés postraumático, rasgos negativistas-desafiantes, trastorno depresivo

\section{DISCUSIÓN}

La psicopatología se encarga del estudio de las enfermedades mentales o de la manifestación de comportamientos y experiencias que pueden ser indicativos de enfermedad mental $o$ deterioro psicológico (16), dividiendo a la mente en varias funciones psíquicas superiores con el fin de facilitar su exploración, estudio y comprensión.

Esa "exploración psicopatológica" (también conocida como examen psíquico o examen del estado mental) es diferente a cualquier otra evaluación en la Medicina, pues se sostiene en enfoques científicos y humanísticos para comprender al paciente. Aunque es parte esencial, pivotante, de la práctica clínica psiquiátrica, todavía existe muy poca información práctica sobre cómo debe enseñarse (16).

El empleo del cine con propósitos educativos cuenta con experiencias positivas en los diferentes niveles de enseñanza, desde la primaria y secundaria hasta la universitaria. En esta última, el cine tiene aplicaciones específicas en carreras vinculadas a la salud, como Medicina o Psicología (17).

En Psiquiatría, las películas pueden ofrecer descripciones realistas de las características de los personajes, de la presencia de trastornos psicopatológicos, así como de las dinámicas personales y familiares, con enfoque en las normas sociales imperantes. Diversas alteraciones psicopatológicas o alteraciones del estado mental (por ejemplo, fenómenos alucinatorios, ideas delirantes, entre otros) pueden ilustrarse y entenderse a partir de la visualización de escenas de películas. Una ventaja de utilizar películas para la enseñanza es que no se generan preocupaciones sobre la confidencialidad, ni conflictos éticos. Asimismo, los personajes experimentan sus síntomas en el contexto de sus vidas y no en un entorno médico aislado (2).

Universo Servilleta nos presenta la historia de Félix, un joven de veintitantos que se encuentra en la búsqueda de respuestas a los grandes misterios de la vida, la trascendencia y el amor. Félix pierde simultáneamente su trabajo y su novia, quien decide ir a vivir al extranjero en busca de mejores oportunidades. Se refugia en sus mejores amigos, los ricos, pero algo perdidos, Sancho y Eme. Durante la película podemos observar que la hermana de Sancho, presenta síntomas compatibles con un trastorno depresivo, pudiendo observarse reiterados intentos de suicidio.

Cuchillo de Palo se centra sobre la muerte de Rodolfo, un hombre adulto para quien la privacidad resultaba muy importante. Luego de su fallecimiento, su sobrina 
Renate, que lo recordaba cariñosamente como alguien alegre, se pregunta sobre la verdad detrás de su misterioso y distante tío, indagando sobre su vida. De la mano de sus familiares y amigos, se va desentrañando una historia llena de sufrimiento, odio, supresión, ignorancia y miedo, recreándose la vida de Rodolfo desde los ojos de los que alguna vez lo conocieron. Cuchillo de Palo narra poderosamente una historia de discriminación hacia la población LGBT+. Se observan trastornos por traumas, depresivos y de personalidad, en consonancia con el ambiente de terror y de tortura que asoló el país durante los años amargos de la dictadura del general Alfredo Stroessner.

7 cajas nos muestra a Víctor, quien recibe una propuesta inusual para transportar siete cajas de contenido desconocido, a través del Mercado 4 de la ciudad de Asunción, a cambio de dinero. Acepta esta empresa debido a problemas económicos y así, a través de la poderosa trama, se puede sentir la adrenalina del protagonista en su recorrido por los angostos pasillos de ese mercado. En la película se evidencian distintas psicopatologías en el entorno cercano a Víctor, como trastornos del lenguaje $y$, también, alteraciones del control de los impulsos y de la conducta.

La Enamorada pone de relieve la vida de Victoria, una chica sin trabajo que se dedica a vender hierbas medicinales ("yuyos"), que se ve angustiada por una vida llena de soledad y de acoso por parte de los hombres con los que se cruzaba. Al conseguir trabajo en la casa del Dr. Juan Bautista, comienza a sentir amor por él y sufre por este, al enterarse de que se había casado. En el transcurso de la trama se observan varias alteraciones psicopatológicas en Victoria, consumida por problemas de soledad y falta de comunicación con el entorno.

A través de Latas Vacías observamos a Alfonso, un buscador de "plata yvyguy" (tesoros ocultos, enterrados en Paraguay durante la Guerra de la Triple Alianza, que involucró a Paraguay, Brasil, Argentina y Uruguay), quien es golpeado por la tragedia. Huye del campo y de su doloroso pasado para refugiarse en la ciudad de Coronel Oviedo, donde sobrevive trabajando en un vertedero. En la película se puede observar la presencia de alucinaciones visuales, desencadenadas por creencias sobrenaturales sobre la "plata yvyguy", así como ideas delirantes (por ejemplo, de referencia).

En La Chiperita se nos muestra una historia de amor. La trama se complica con dificultades económicas de la familia de la protagonista, que hace que ella tenga que dejar de lado la ilusión del amor para poder ayudar a su familia. A lo largo de la cinta destaca la presencia de síntomas compatibles con ansiedad patológica.

Los Buscadores cuenta la historia de Manu que junto con Fito y Don Elio están tras las pistas de un supuesto tesoro de la época de la Guerra Grande (Triple Alianza) o "plata yvyguy", que está enterrado en los jardines de la residencia de un embajador. En la trama se encuentran elementos de la idiosincrasia paraguaya, una familia extensa en torno al protagonista, la creencia fantástica en todas las leyendas que giran en torno al botín, así como el uso del "jopara" (forma coloquial del guaraní hablado en Paraguay, que utiliza una serie de palabras prestadas en español). La película también muestra la diferencia de clases y la desidia del Estado. En el largometraje se observan a personas de condición social vulnerable que desean encontrar el tesoro para beneficio propio o para ayudar a la familia. Se observan síntomas delirantes, ansiedad y violencia.

Leal, solo hay una forma de vivir nos narra una historia en donde, luego de fallidos intentos por detener el narcotráfico, se forma un grupo paramilitar liderado por un ex coronel. En el filme se observan trastornos relacionados muchas veces con el perfil de narcotraficantes y sicarios, tales como rasgos de personalidad narcisista y de personalidad antisocial, así como trastornos adictivos.

En Las Herederas se puede visualizar a dos mujeres que habían heredado lo suficiente como para vivir cómodamente sin necesidad de trabajar, pero cuando ambas tienen más de sesenta años, el dinero se acaba. Una de ellas va a la cárcel y la otra comienza trabajar, ofreciendo un servicio de taxi a otras mujeres. Es así como conoce a una mujer 20 años menor, quien le genera una revolución interna. En la cinta se observa que los problemas económicos llevan a una de las protagonistas a enfrentarse con la depresión, quien además padece de trastorno obsesivo-compulsivo.

Por último, El Supremo Manuscrito se centra en la búsqueda del libro Yo El Supremo, de Augusto Roa Bastos. El manuscrito desaparece durante una millonaria subasta. El rematador, Anton Remianiuk, y la ganadora de la subasta, Ana Morel, se enfrentan a numerosos peligros con el afán de encontrar la valiosa obra literaria. Pedro, quien sufre de estrés postraumático y elementos negativistas-desafiantes, es cómplice del robo del manuscrito, pero luego es traicionado y finalmente ayuda a los protagonistas a encontrarlo. Sin embargo, también busca venganza y termina matando a quien lo traicionó. Además del estrés postraumático y de las alteraciones negativistasdesafiantes, en la película también se observan signos y síntomas del trastorno de depresión mayor en la joven Rufina, quien sufrió maltratos y explotación 
desde pequeña.

Investigadores han afirmado que el cine es una herramienta valiosa para el estudio de situaciones vitales trascendentales, tales como el dolor, la enfermedad y la muerte (17-19). En el caso específico de la Psiquiatría, diversos investigadores han afirmado que el cine es útil para su enseñanza (20-22), tanto a estudiantes de Medicina como a médicos en formación. Por ejemplo, un estudio realizado en América Latina ha informado resultados positivos y los estudiantes participantes han expresado que, a través de las películas y con la guía del docente, la identificación de síntomas y síndromes psiquiátricos, así como sus situaciones causales, les ha sido fácil de realizar y que esta manera de estudiar Psiquiatría es enriquecedora (17).

Las películas representan un instrumento de aprendizaje único y agradable, que puede generar debates y ofrecer una forma de descubrir lo que piensan los estudiantes sobre temas que de otro modo permanecerían ocultos o fácilmente eclipsados por cuestiones técnicas médicas (23-25). Las películas se pueden utilizar con éxito para señalar varios aspectos de las condiciones psiquiátricas que aquejan a las personas. A través de ellas, los estudiantes pueden aprender complejas realidades de los aspectos personales y sociales de las personas con trastornos mentales y comprender mejor las perspectivas de las mismas. La ventaja de usar películas para enseñar en lugar de las clásicas conferencias o clases teóricas es que se puede ver en lugar de simplemente escuchar sobre psicopatología y que la información importante se presenta en forma condensada en una película (26). Asimismo, otros investigadores han reportado que enseñar con películas es una herramienta poderosa para desarrollar características y comportamientos positivos en los jóvenes (27). Por ejemplo, la película 7 cajas es una de las 36 películas que han sido distribuidas a 9000 escuelas secundarias, además de institutos superiores de formación docente, de la República Argentina, en el marco del Archivo Fílmico Pedagógico "Jóvenes y Escuelas" del Ministerio de Educación argentino. La idea de este archivo fílmico es promover debates sobre discriminación, sexualidad o trabajo (28).

Una desventaja de usar películas para la enseñanza de la Psiquiatría puede ser la distorsión y la imagen estigmatizante de la enfermedad mental (2), por lo que la selección de las películas debe hacerse por docentes con experiencia y siguiendo criterios establecidos por los objetivos de enseñanza.

El cine paraguayo, cada vez más rico y diverso, además de ser una fuente de inspiración y entretenimiento, puede ayudar en la enseñanza de la Medicina en general, y de la Psiquiatría en particular. A través de sus elaboradas historias, el cine paraguayo puede ayudar en la construcción de una Medicina más humanista, aumentar la motivación, el compromiso y el aprendizaje de los estudiantes de Medicina y los médicos jóvenes (29-31), así como fortalecer el profesionalismo, la ética y las habilidades de comunicación $(32,33)$.

La principal fortaleza de este trabajo es que, hasta el límite de nuestros conocimientos, es el primer estudio realizado en Paraguay que buscó determinar la presencia de algún tipo de psicopatología en películas paraguayas, desplegando así innumerables posibilidades de mayor investigación en el área. La principal limitación de este trabajo es el reducido tamaño muestral y que los resultados referentes a la psicopatología encontrada no hayan sido validados por expertos ajenos al equipo de investigación.

\section{AGRADECIMIENTOS}

Los autores agradecen a Tana Schémbori y a Juan Carlos Maneglia, productores y cineastas paraguayos, por la retroalimentación brindada en el desarrollo de este artículo

\section{REFERENCIAS}

1. Stucchi-Portocarrero S, Herrera-Lopez V. Cine peruano actual y psicopatología. Rev Neuropsiquiatr. 2014;77(4):207-213. URL.

2. Bhugra D. Teaching psychiatry through cinema. Psychiatric Bulletin 2003;27(11):429-430.

https://doi.org/10.1192/pb.27.11.429

3. Akram A, O'Brien A, O'Neill A, Latham R. Crossing the line-learning psychiatry at the movies. Int Rev Psychiatry. 2009;21(3):267-268.

https://doi.org/10.1080/09540260902746880

4. Castaldelli-Maia JM, Castilho Mancini S, Castaldelli-Maia M, Lotufo Neto F. Psicopatologia no cinema brasileiro: um estudo introdutório. Rev. psiquiatr. clín. 2005;32(6):319323. https://doi.org/10.1590/S0101-60832005000600002

5. American Psychiatric Association. Diagnostic and Statistical Manual of Mental Disorders. 5th ed. Arlington, VA: American Psychiatric Publishing; 2013.

6. IMDb. Universo Servilleta [Internet]. IMDb. 2020 [citado el 31 de octubre de 2020]. Disponible en: https://www.imdb.com/title/tt1743259/?ref =fn al tt 1

7. IMDb. Cuchillo de Palo [Internet]. IMDb. 2020 [citado el 31 de octubre de 2020]. Disponible en: https://www.imdb.com/title/tt1641619/?ref =fn al tt 1

8. IMDb. 7 cajas [Internet]. IMDb. 2020 [citado el 31 de octubre de 2020]. Disponible en: https://www.imdb.com/title/tt2333598/?ref =fn al tt 1

9. IMDb. La Enamorada [Internet]. IMDb. 2020 [citado el 31 de octubre de 2020]. Disponible en: https://www.imdb.com/title/tt2338111/?ref =nv sr srsg o

10. IMDb. Latas Vacías [Internet]. IMDb. 2020 [citado el 31 de 
octubre de 2020]. Disponible en: https://www.imdb.com/title/tt4319490/?ref =fn al tt 1

11. IMDb. La Chiperita [Internet]. IMDb. 2020 [citado el 31 de octubre de 2020]. Disponible en: https://www.imdb.com/title/tt5052792/?ref =nv sr srsg $\underline{0}$

12. IMDb. Los Buscadores [Internet]. IMDb. 2020 [citado el 31 de octubre de 2020]. Disponible en: https://www.imdb.com/title/tt4912622/?ref =fn al tt 1

13. IMDb. Leal, solo hay una forma de vivir [Internet]. IMDb. 2020 [citado el 31 de octubre de 2020]. Disponible en: https://www.imdb.com/title/tt7606620/?ref =fn al tt 1

14. IMDb. Las Herederas [Internet]. IMDb. 2020 [citado el 31 de octubre de 2020]. Disponible en: https://www.imdb.com/title/tt7875464/?ref =nv sr srsg $\underline{0}$

15. IMDb. El Supremo Manuscrito [Internet]. IMDb. 2020 [citado el 31 de octubre de 2020]. Disponible en: https://www.imdb.com/title/tt9055158/?ref $=\mathrm{fn}$ al tt 1

16. Arce A, Torales J, Gómez N, Maggi C, editores. La exploración psicopatológica: una introducción a la semiología psiquiátrica. 1ạ ed. Asunción: EFACIM; 2017.

17. Hernández Figaredo P, Peña García F. El cine como herramienta en la docencia de Psiquiatría. Rev Hum Med. 2013;13(1):244-265. URL.

18. Astudillo Alarcón W, Mendinueta Aguirre C. El cine en la docencia de la medicina: cuidados paliativos y bioética. Rev. Medicina y cine. 3(1):32-41. URL.

19. Arreaza E, Sulbarán E, Ávila R. Aplicación de una guía didáctica sobre cine para generar conocimiento en educación: resultados preliminares. Rev Enlace. 2009;6(2):71-82. URL.

20. Datta V. Madness and the movies: an undergraduate module for medical students. Int Rev Psychiatry. 2009;21(3):261-266. https://doi.org/10.1080/09540260902748001.

21. Dave S, Tandon K. Cinemeducation in psychiatry. Adv Psychiatr Treat. 2011;17(4):301-308. https://doi.org/10.1192/apt.bp.107.004945

22. Jukić $V$, Brecić $P$, Savić A. Movies in education of psychiatry residents. Psychiatr Danub. 2010;22(2):304-307. URL.

23. Fritz GK, Poe RO. The role of a cinema seminar in psychiatric education. Am J Psychiatry. 1979;136(2):207210. https://doi.org/10.1176/ajp.136.2.207

24. Darbyshire D, Baker P. Cinema in Medical Education - Has it Penetrated the Mainstream? Rev Med Cine. 2011;7(1):814. URL.

25. Batistatou A, Doulis EA, Tiniakos D, Anogiannaki A, Charalabopoulos $\mathrm{K}$. The introduction of medical humanities in the undergraduate curriculum of Greek medical schools: challenge and necessity. Hippokratia. 2010;14(4):241-243. URL.

26. Kalra G. Psychiatry movie club: A novel way to teach psychiatry. Indian J Psychiatr. 2011;53(3):258-260. https://doi.org/10.4103/0019-5545.86820

27. Smithikrai C. Effectiveness of Teaching with Movies to Promote Positive Characteristics and Behaviors. Procedia Social and Behavioral Sciences 2016;217:522-530. https://doi.org/10.1016/i.sbspro.2016.02.033

28. Última Hora. "7 Cajas" estará en las aulas de las escuelas argentinas [Internet]. Última Hora. 2015 [citado el 02 de noviembre de 2020]. Disponible en: https://www.ultimahora.com/7-cajas-estara-las-aulas-lasescuelas-argentinas-n893788.html

29. Klemenc-Ketis Z, Kersnik J. Using movies to teach professionalism to medical students. BMC Med Educ. 2011;11(1):60. https://doi.org/10.1186/1472-6920-11-60

30. Blasco PG, Garcia DS, de Benedetto MAC, Moreto G, Roncoletta AF, Troll T. Cinema for educating global doctors: from emotions to reflection, approaching the complexity of the Human Being. PrimaryCare. 2010;10(3): 45-47. URL.

31. Alexander M, Pavlov A, Lenahan P. Lights, camera, action: using film to teach the ACGME competencies. Fam Med. 2007;39(1):20-23. URL.

32. Winter RO, Birnberg BA. Teaching professionalism artfully. Fam Med. 2006;38(3):169-171. URL.

33. Blasco PG, Moreto G, Roncoletta AF, Levites MR, Janaudis MA. Using movie clips to foster learners' reflection: improving education in the affective domain. Fam Med. 2006;38(2):94-96. URL 